\title{
Introduction to the Issue on Advanced Signal Processing for Brain Networks
}

$\mathbf{N}$ ETWORK models of the brain have become an important tool of modern neurosciences to study fundamental organizational principles of brain structure \& function. Their connectivity is captured by the so-called connectome, the complete set of structural and functional links of the network. Advancing current methodology remains an important need in the field; e.g., increasing large-scale models; incorporating multimodal information in multiplex graph models; dealing with dynamical aspects of network models; and matching data-driven and theoretical models.

These challenges form multiple opportunities to develop and adapt emerging signal processing theories and methods at the interface of graph theory, machine learning, applied statistics, simulation, and so on, to play a key role in analysis and modeling and to bring our understanding of brain networks to the next level for key applications in cognitive and clinical neurosciences, including brain-computer interfaces.

We were impressed by the more than 50 high-quality submissions that we received for this special issue. After an extensive peer-review process, 16 excellent papers were selected for inclusion in this issue.

The issue begins with a review article by Silva et al. on the rich field of blind source separation and the applications to unimodal and multimodal neuroimaging data. Different BSS models are revisited and classified according to their underlying assumptions about the sources' mixtures, linearity of mapping, statistical relationships, and so on.

The first series of papers is dedicated to processing of functional magnetic resonance imaging (fMRI) data. The construction of functional networks typically relies upon conventional pairwise Pearson correlation measures between regional timecourses. Ahmad et al. propose a Bayesian approach to connectivity estimation, which has the advantage of providing built-in estimates of the uncertainty of connectivity estimates. Because the fMRI BOLD signal is inherently noisy and inter-individual differences are significant, this type of approach offers a principled way of overcoming the shortcomings of point estimates. In order to analyse brain networks with a combination of clustering models and component decompositions, Hinrich et al. extend archetypal analysis to multi-subject fMRI data. Both spatial and temporal archetypal analyses are explored, which lead to brain parcellations and temporal dynamics of functional networks, respectively. Liu et al. formulate a novel sparse regression that simultaneously models both stationary (i.e., measured over the whole resting-state session) and dynamic (i.e., using sliding-window approach) functional connectivity components of a neural system. An accelerated gradient method is applied to determine the most relevant stationary and dynamic components. They demonstrate the utility of their method in identifying abnormalities in functional connectivity in Parkinsonian patients. A potential limitation of their approach is the directed nature of the inferred connectivity measurements. As statistical

Digital Object Identifier 10.1109/JSTSP.2016.2602945 inference is an essential step to assess brain networks, Zhan et al. enhance the original network-based statistic with various nodal parcellation atlases and test statistics. They demonstrate the use of this novel extension in identifying differentially expressed functional subnetworks in individuals with mild cognitive impairment and Alzheimer's disease. In particular, the authors identify a parametric decrease in resting-state functional connectivity between these two cohorts and a healthy control group. In work by Huang et al., graph signal processing is developed by considering fMRI activity timecourses on the backbone of a functional-connectivity network. Using the spectral approach, they generalize several signal-processing operations such as filtering and total-variation regularization. Multimodal brain network mapping methods that combine different imaging modalities and resolutions have gained significant interest in recent years. Rahim et al. propose a regularized regression model for region-to-voxel functional connectivity estimation in which the connectivity estimates for each voxel are weighted according to a prior on metabolic activity. This prior is inferred from fluorodeoxyglucose positron emission tomography (FDGPET) and assumes that functional connectivity is coupled with metabolic activity. The correctness of this assumption remains to be validated. Using their multimodal approach, the authors were able to discriminate between patients with Alzheimer's disease and healthy controls with accuracies that ranged between $70-80 \%$.

Finally, Hu et al. explore white-matter structural connectivity to investigate the spread of brain pathology in neurological disorders; i.e., brain networks provide conduits for pathology to spread with disease progression to unaffected grey matter regions. In particular, they develop a brain network diffusion model to predict the spatial progression of grey matter atrophy in Alzheimer's disease. The connections in the diffusion model are estimated with automated fibre tractography. The authors specifically focus on using their model to identify the "sources" of atrophy, which is achieved with sparse regularization on the source distribution and solution of the inverse problem with gradient descent. Further work is needed to assess the utility of the diffusion model in longitudinal data.

The second series of papers considers the extraction and analysis of brain networks from electrophysiology data. Andalibi et al. present algorithms for the computation of a particular functional connectivity analysis based on the Cox method and achieve a speed up by a factor of 100 when parallelized on Graphical Processing Units (GPUs). They validate their method on neuronal recordings, which also opens up exciting possibilities for other applications such as in time-variant analyses of connectivity serving as biomarkers of disorders and cognitive aging. Mishne et al. go a step further and explore the hidden connectivity patterns at multiple time scales. They accomplished extraction of neuronal activity patterns by applying a hierarchical coupled geometry analysis, which provides local to global representations through a novel multiscale metric. Weichwald et al. describe MERLiN (Mixture Effect Recovery in Linear Networks), a significant extension of linear 
mediation analysis with which they construct causal variables from observed, multivariate non-causal variables as a manifold optimization problem. They demonstrate the utility of their approach with EEG data, describe its potential extension to other modalities, and provide their software on Github. Malladi et al. apply the concepts of functional and causal connectivity to a concrete clinical setting, that is the identification of the seizure onset zone. Their approach is based on directed information, which allows the construction of a causal graph between electrocorticographic $(\mathrm{ECoG})$ brain data of patients and the subsequent successful estimation of the seizure onset zone. Gonuguntla et al. develop a novel framework for the classification of subject and task specific functional networks. As functional connectivity can vary over time, computational analysis of functional connectivity may be very time consuming. Higashi et al. address the problem of improving classification accuracy in Brain Computer Interfaces using event-related potentials. By using a new supervised multi-linear discriminant analysis (MLDA) and carefully regularizing the parameter subspaces with constraints that incorporate measures of brain function connectivity they show that it is possible to improve single-trial classification accuracy against a broad range of alternate MLDA approaches. Monajemi et al. couple brain connectivity from EEG with gait measures for Parkinson disease patients with different levels of movement tremor. They apply a novel adaptive multi-task diffusion approach to estimate an underlying model between gait and EEG signals by coupling EEG connectivity measures with distributed learning strategies over adaptive networks. Finally, Wang et al. propose to use a vector autoregressive model in source space for analysis of EEG data. This is a workable and interpretable approach for low-dimensional analysis of directed functional connectivity in resting-state EEG, and is applied here to show interesting relationship with regions implicated in fMRI resting-state networks.

We hope that this Issue will be stimulating and inspiring to readers. We thank the reviewers for their time and effort in carefully reviewing submissions to ensure a special issue of the highest quality. We also thank the JSTSP Editorial Board and staff for their assistance and guidance throughout this process.

\section{VAN DE VILlE, Lead Guest Editor}

Ecole Polytechnique Fédérale de Lausanne

University of Geneva

Geneva CH-1211, Switzerland

dimitri.vandeville@epfl.ch

V. JIRSA, Guest Editor

Institut de Neuroscience des Systèmes UMR1106 Inserm Aix-Marseille University

Marseille 13005, France

viktor.jirsa@univ-amu.fr

S. STROTHER, Guest Editor

Rotman Research Institute,

Baycrest and University of Toronto

Toronto, ON M5S 1A1, Canada

sstrother@research.baycrest.org

J. RICHIARDI, Guest Editor

University of Geneva

Geneva CH-1211, Switzerland

jonas.richiardi@unige.ch

A. ZALESKY, Guest Editor
The University of Melbourne
Melbourne, Vic 3010, Australia
azalesky@unimelb.edu.au

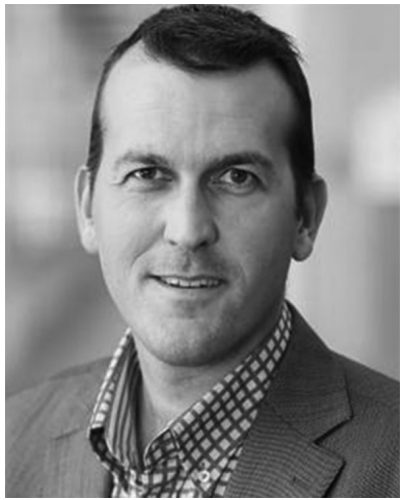

Dimitri Van De Ville (M'02-SM'12) received the M.S. degree in engineering and computer sciences and the Ph.D. degree both from Ghent University, Ghent, Belgium, in 1998, and 2002, respectively.

After a postdoctoral stay (2002-2005) in the lab of Prof. M. Unser at the Ecole Polytechnique Fédérale de Lausanne (EPFL), Switzerland, he became responsible for the Signal Processing Unit, University Hospital of Geneva, Switzerland, as part of the Centre d'Imagerie Biomédicale. In 2009, he received a Swiss National Science Foundation professorship and since 2015 became a Professor of bioengineering at the EPFL and the University of Geneva, Switzerland. His research interests include wavelets, sparsity, pattern recognition, and their applications in computational neuroimaging.

Dr. Van De Ville served as an Associate Editor for the IEEE TRANSACTIONS ON IMAGE Processing from 2006 to 2009 and the IEEE SignAL PROCESSING LETTERS from 2004 to 2006. He was the Chair of the Bio Imaging and Signal Processing TC of the IEEE Signal Processing Society from 2012 to 2013 . He is the Co-Chair of the biennial Wavelets \& Sparsity series conferences, together with V. Goyal and M. Papadakis. He received the Pfizer Research Award 2012, the NARSAD Independent Investigator Award 2014, and the Leenaards Foundation Award 2016.

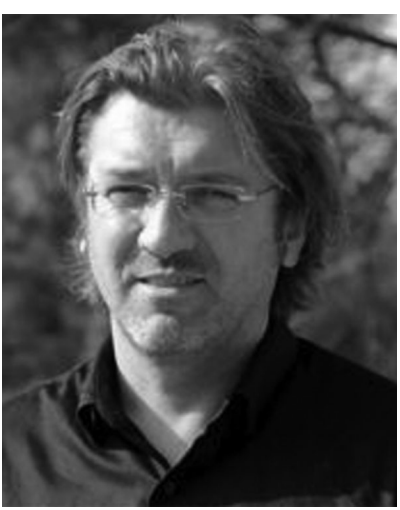

Viktor Jirsa received the M.S. degree in elementary particle physics from Victoria University, Manchester, U.K., in 1991, the Diploma degree in physics, in 1994, and the Ph.D. degree in theoretical physics, in 1996, both from the University of Stuttgart, Stuttgart, Germany. In 19961999, he was a Research Fellow (Deutsche Forschungsgemeinschaft and Human Frontiers Science Fellow) at the Center for Complex Systems \& Brain Sciences, Florida Atlantic University, then became an Assistant Professor during 1999-2004, and an Associate Professor during 2004-2005 at the Center and the Physics Department, Florida Atlantic University. In 2006, he joined the Centre National de la Recherche Scientifique (CNRS) in Marseille, France, as the Director of Research, where he still is. In 2012, he founded the Institute of System Neuroscience with Patrick Chauvel at Aix-Marseille University and became its Director in 2014. His research interests include large-scale modeling of brain network dynamics, spatiotemporal organization, and nonlinear dynamic phenomena. In the last ten years, he emphasized the neuroinformatics of personalized biologically realistic brain network models and their clinical applications. He serves 
on various editorial and scientific advisory boards including Journal of Mathematical Neuroscience and Scientific Reports, and is Section-Editor-in-Chief of the European Physical Journal and Nonlinear Biomedical Physics. He received various national and international awards including the Francois Erbsman Prize in 2001 and the Scientific Excellence Award of CNRS in 2011.

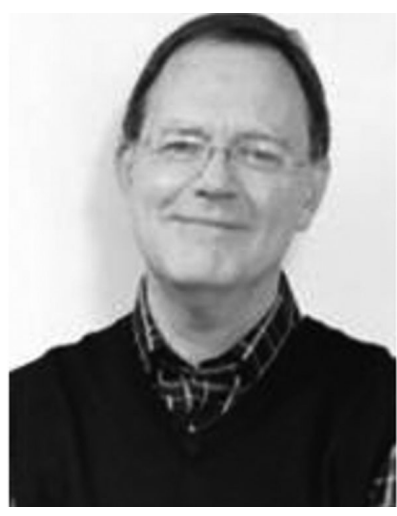

Stephen Strother received the M.S. (Hons) degree in physics and mathematics at Auckland University, Auckland, New Zealand, in 1979, and the Ph.D. degree in electrical engineering from McGill University, Montreal, Canada, in 1986. After a PET fellowship at Memorial Sloan Kettering Cancer Center, New York, in 1989, he joined the VA Medical Center, Minneapolis as a Senior PET Physicist, and a Professor of radiology at the University of Minnesota. In 2004, he became a Senior Scientist at the Rotman Research Institute, Baycrest, and a Professor of medical biophysics at the University of Toronto. He leads the group building the neuroimaging component of the BrainCODE data infrastructure for the Ontario Brain Institute (OBI), and co-leads neuroinformatics/neuroimaging platforms for several OBI integrated disease discovery programs. His research interests include neuroinformatics and data reproducibility with a focus on optimization of PET, fMRI/MRI and EEG neuroimaging pipelines using statistical/machine-learning techniques. He was the Chairman of the International Neuroinformatics Standards Committee, National Institutes of Health, USA that developed the NIfTI data format standard for neuroimaging, and is an Associate Editor for Human Brain Mapping and Frontiers in Neuroscience: Brain Imaging Methods. He is also a Cofounder of Predictek, Inc., and ADMdx in Chicago, medical analysis and diagnostics companies.

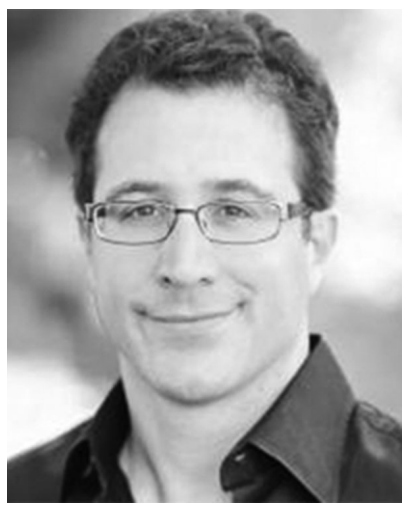

Jonas Richiardi (M'05) received the B.Eng. degree in electronic engineering at the University of Essex, Colchester, U.K., in 2001, the M.Phil. degree in computer speech, text, and internet technology from the University of Cambridge (Darwin College), Cambridge, U.K., in 2002, and the Ph.D. degree in signal processing and pattern recognition from the Ecole Polytechnique Fédérale de Lausanne (EPFL) (Laboratory of IDIAP, Signal Processing Institute), Lausanne, Switzerland, in 2007. He then co-founded the PatternLab, an engineering and scientific consulting company, and returned to academia as a Postdoctoral Fellow in 2009 in Dimitri Van de Ville's Medical Image Processing Laboratory at EPFL. He received the Marie Curie Fellowship and spent two years at Stanford before coming back to Switzerland (University of Geneva).

Dr. Richiardi started the Pattern Recognition in NeuroImaging workshop series in 2011, and has served as a Programme Chair, a Finance Chair, a Steering Committee Chair, and a Member. He serves as a Member and the Web Liaison of the IEEE Signal Processing Society's Technical Committee on Bio Imaging and Signal Processing.

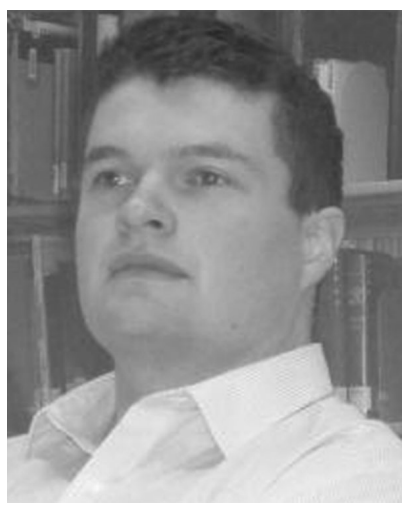

Andrew Zalesky received the Ph.D. degree from the Department of Electrical and Electronic Engineering, University of Melbourne, Melbourne, Australia, in 2006. He is currently working with neuroscientists, utilizing his engineering expertise in networks to understand human brain organization in health and disease. He has developed widely used methods for modeling and performing statistical inference on brain imaging data. His methods are utilized to investigate brain connectivity abnormalities in disease. He identified some of the first evidence of connectome pathology in schizophrenia. He currently holds a fellowship from the National Health and Medical Research Council of Australia. He is based at the University of Melbourne and holds a joint appointment between the Melbourne Neuropsychiatry Centre and the Melbourne School of Engineering. He leads the Systems Neuropsychiatry Group. In 2016, he co-authored the first textbook on connectomics. He has published in a diverse range of fields, including optics, statistics, neuroimaging and schizophrenia. He serves on the editorial boards of NeuroImage, Brain Topography, and Network Neuroscience. He received the Young Tall Poppy Science Award in 2014 and the Aubery Lewis Award in 2016. 